\author{
Chemistry \\ Platform of the Swiss Academy of Sciences
}

\section{The YFM 2012 - Emerging Research and Established Needs: Fund Raising, Publishing and Career Advancement}

\section{Barbara Winter-Werner*a, Franziska Schoenebeck ${ }^{* b}$, and Ryan Gilmour ${ }^{\star b}$}

${ }^{\star}$ Correspondence: Dr. B. Winter-Wernera ${ }^{a}$, Prof. Dr. F. Schoenebeck ${ }^{\text {, }}$ Prof. Dr. R. Gilmour

aSwiss Academy of Sciences (SCNAT), Platform Chemistry, Schwarztorstrasse 9, CH-3007 Bern, E-mail: barbara.winter@scnat.ch

${ }^{b}$ ETH Swiss Federal Institute of Technology, Laboratory of Organic Chemistry, Wolfgang-Pauli-Str. 10, CH-8093 Zürich, E-mail: schoenebeck@org.chem.ethz.ch ryan.gilmour@org.chem.ethz.ch

The fifth Young Faculty Meeting (YFM) took place on June 7, 2012 at the University of Bern and brought together again almost thirty young researchers from all Swiss universities and both federal institutes of technology. This yearly symposium was organized by the «Platform Chemistry» of the Swiss Academy of Sciences (SCNAT) together with Franziska Schoenebeck (ETH Zurich) and Felix Zelder (University of Zurich) and generously supported by the Kontaktgruppe für Forschungsfragen $\mathbf{K G F}$ (www.kgf.ch).

Following the opening remarks by Franziska Schoenebeck (ETH Zürich), the morning session got underway with a series of scientific presentations by young academics. The first lecture by Florian Seebeck (University of Basel), described his group's work on biosynthetic sulfur transfers, with major emphasis placed on the example of ergothioneine, which is a histidinederived thiol of bacterial and fungal origin which has also been isolated from animal and human tissue. Recent findings indicate that ergothioneine plays a crucial role in human physiology, but its role in microbial life is poorly understood. Seebeck and his co-workers have identified a five gene cluster from mycobacteria which is responsible for ergothioneine biosynthesis. The key reaction is catalyzed by an unusual non-heme iron enzyme which affords oxidative insertion of a sulfur atom into the imidazole $\mathrm{C}(2)-\mathrm{H}$ bond of histidine. The Basel team is now investigating the catalytic mechanism of this unprecedented enzymatic reaction. The second speaker of the morning session, Gilles Gasser (University of Zurich), started his lecture by explaining the potential advantages of using metal complexes over purely organic compounds for the development of novel anticancer agents. He reminded the audience that many metal-based drugs were already on the market and that several others were in clinical trials with promising preliminary results. He then presented the latest results of his group on the preparation of easy-to-synthesize, anti-proliferative $\mathrm{Ru}(\mathrm{II})$ complexes. The most potent of these compounds to date has already been subject to further biological investigations and was found to have a relatively unique mode of action. His group is now working on specifically delivering his lead compound to cancer cells. Afterwards, Ján Cvengroš (ETH Zurich) presented his work on the synthesis and applications of $\mathrm{N}$-stereogenic molecules. He first gave a brief introduction of Tröger's base, a molecule possessing stable stereogenic nitrogen atoms. Since the supply of enantiomerically pure Tröger's base analogues is rather limited, his group focuses on the development of efficient synthetic methods for their preparation in enantiomerically pure form. He also demonstrated that the Tröger's base scaffold can be easily used as a backbone for a variety of ligands which can be efficiently applied in catalysis (e.g. for $\mathrm{C}-\mathrm{C}$ cross-coupling reactions).

In the second half of the morning session, Karl Gademann (University of Basel) and Roland Sigel (University of Zürich) presented very candid overviews of their respective careers to date. In addition to touching on a number of pertinent topics, including mentorship, career planning and funding opportunities, both speakers were happy to take more specific questions from the audience. Discussions regarding the relative merits of the habilitation versus the assistant professorship in Switzerland were particularly engaging, particularly as Professor Gademann has experienced both systems. This discussion naturally touched upon the tenure/non-tenure systems that are often institutiondependent, and the new initiatives, such as the Ambizione fellowship, that has been introduced. In general, the multitude of possibilities for young scientists to start their careers in Switzerland was deemed to be a positive development; however, the lack of 'landing places' for the ever-increasing number of junior faculty was seen as concerning. Both guest speakers reflected on their careers in a very personal manner, stressing the importance of mentorship as well as visibility (presentation at conferences) as key factors for development. Funding opportunities (SNF, COST, local support, fellowships) were raised, and group supervision and management strategies proved a popular topic of debate. A fruitful and interactive discussion followed these excellent presentations.

After an enjoyable lunch, the first speaker of the afternoon session, Hans Jakob Wörner (ETH Zürich), reported on the recent progress of his group in measuring electronic dynamics in molecules on time scales of femtoseconds ( $1 \mathrm{fs}=10^{-15} \mathrm{~s}$ ) to attoseconds ( 1 as $\left.=10^{-18} \mathrm{~s}\right)$. He showed how the process of attosecond pulse generation gives insight into the electronic structure of molecules, its evolution in the course of a photochemical reaction on the femtosecond time scale and into the rearrangement of electrons on the attosecond time scale. His group recently succeeded in performing such experiments in an ensemble of molecules that had a well-defined orientation in space for the duration of the measurement, giving access to the finer details of electronic structure and dynamics. This lecture was followed by a presentation on exploring the mechanisms and evolution of natural enzymes by Marc Creus (University of Basel), who aims to learn about the fundamental principles of enzyme chemistry. To illustrate the approach, he presented studies on the emergence of a new activity in a bacterial metalloenzyme, which arises from simple replacement of a $\mathrm{Zn}$ in the catalytic site to a $\mathrm{Mn}$; this replacement leads the desuccinylase to acquire a dipeptidase activity. This research may also have useful practical applications, particularly as the enzyme under investigation is a potential target for novel antibiotics. Moreover, the general insights gained from laboratory evolution may prove useful for the design of novel biocatalysts. Last but not least Henning Jessen (University of Zurich) introduced densely phosphorylated diphospho-inositol-polyphosphates as important secondary messengers in living organisms. As these highly charged and unstable natural products are not readily obtained in good purity and reasonable amounts by extraction from biological sources, 

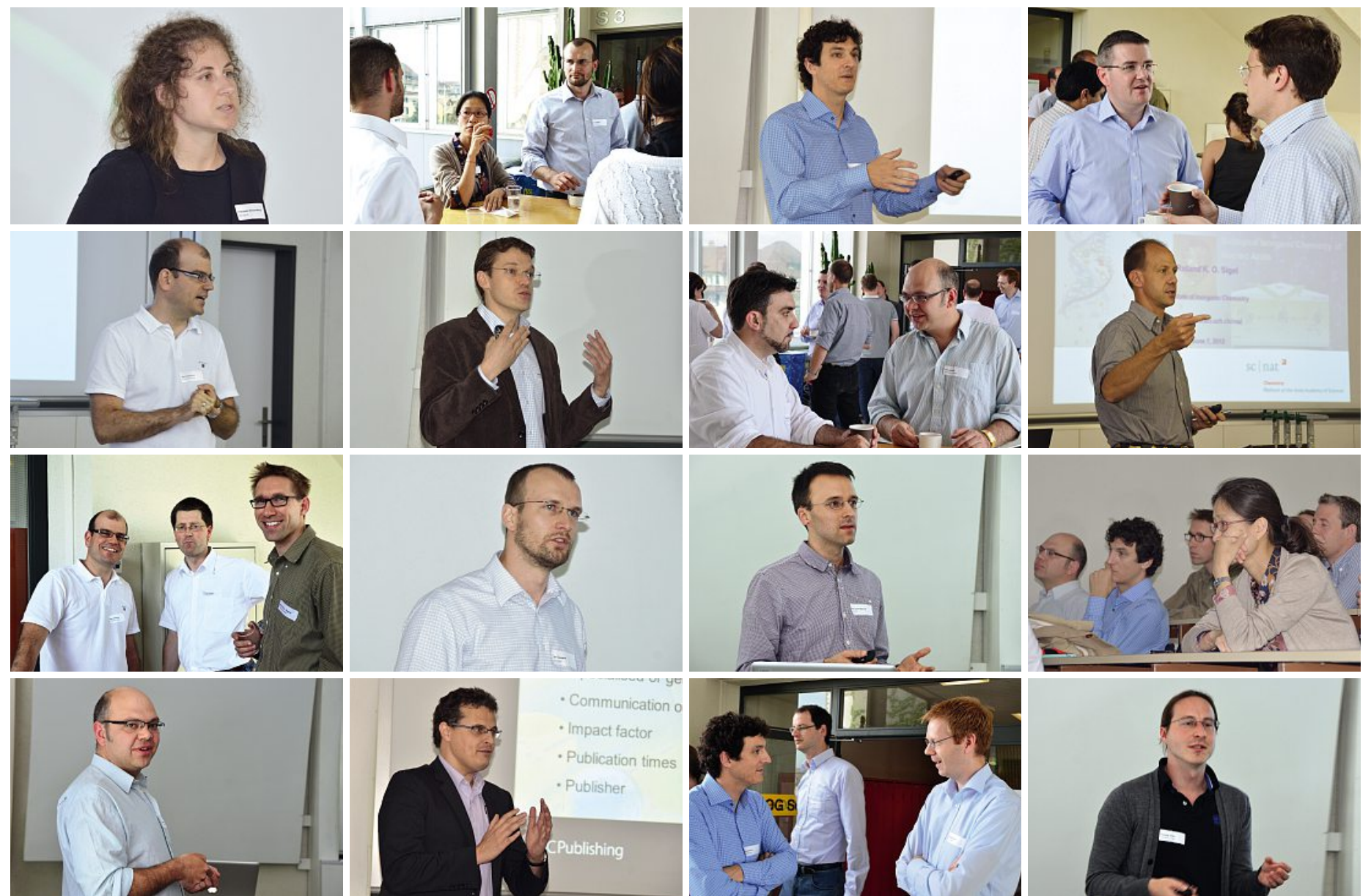

Impressions of the «Young Faculty Meeting 2012»

a unified chemical total synthesis has been designed. It was demonstrated that the synthetic route could be efficiently adapted to obtain all possible stereoisomers of the diphospho-inositolpolyphosphates in high yield, purity and enantiomeric excess, thus paving the way for detailed biological studies.

In the final session of the day, Javier Pérez-Ramirez (ETH Zürich) gave a stimulating and highly entertaining presentation on 'Publishing - from the editor's point of view'. Prof. PérezRamírez is currently an associate editor of the journal Catalysis Science \& Technology from the Royal Society of Chemistry. He discussed the advantages and disadvantages of various editorial approaches (academic versus non-academic editorial offices or mixtures thereof) and the mechanisms and strategies of the journals with which he has been associated. Naturally, the audience posed a number of specific questions to Professor Pérez-Ramírez ranging from how to prepare a cover letter through to dealing with reviewer and editor comments. The ensuing discussion was extremely lively and continued into the early evening! The three guest speakers are gratefully acknowledged for kindly taking time from their busy schedules to participate in this meeting.

\section{Acknowledgements}

We would like to thank for the financial support of the Kontaktgruppe für Forschungsfragen KGF (www.kgf.ch) and the SCNAT (www.scnat. ch). 